\title{
Deposits of the hydrocarbon raw materials of the Republic of Kazakhstan, where it is possible to introduce a microbiological method for stimulating the formation
}

\author{
Olga Kuderinova ${ }^{1, *}$, Makhambet Shmanov ${ }^{1}$, and Mykhailo Filatiev ${ }^{2}$ \\ ${ }^{1}$ Karaganda Technical University, Development of Mineral Deposits Department, 56 N. Nazarbayev Str., 100027, Karaganda, Republic \\ of Kazakhstan \\ ${ }^{2}$ Volodymyr Dahl East Ukrainian National University, 59a Central Ave., Severodonetsk, 93406, Ukraine
}

\begin{abstract}
The article is devoted to the analysis and systematization of data on hydrocarbon deposits in the Republic of Kazakhstan, highlighting those aspects that will allow the use of this method. The microbiological method of exposure refers to the chemical methods of the tertiary stage of development of oil reservoirs. It has already proven its effectiveness in highly depleted, waterflooded formations with irregular, diffuse oil saturation. Its main advantages are its relative cheapness, it does not require additional equipment during injecting of the microorganisms into the reservoir, and for their nutrition, as a rule, food industry waste is used, and its implementation cannot cause harmful effects on the environment. Also, a fairly extensive database was compiled, according to which various classifications of the republic's deposits were created.
\end{abstract}

\section{Introduction}

The purpose of the study was to identify the parameters needed for the introduction of the method of microbiological influence on oil reservoirs, justification of the need for its use, as well as collection, analysis and systematization of data on all known this type deposits of the republic.

Nowadays, when the reserves-to-production ratio is declining in all countries, which are advanced in oil production, the introduction of methods of improving oil recovery is more urgent than ever before.

Well-known are thermal (steam, hot water, formation combustion are used), gas (carbon dioxide, air, natural gas, nitrogen, flue gases, etc. are injected directly into the formation), chemical (oil is displaced by using surfactants, polymers, alkalis, acids and other reagents), hydrodynamic (water is injected), physical (using wave and electromagnetic radiation, hydraulic fracturing, drilling horizontal wells) and, naturally, combined methods.

Due to extensive application experience, their disadvantages are also known: when pumping steam and hot water, chemical reagents of various types, gases, equipment quickly wears out; and the use of physical methods initially requires the installation of expensive additional equipment.

In addition, when igniting the formation and using chemicals, it is impossible to avoid harmful effects on the environment. In the first case, combustion is difficult to control and as a result, instead of liquefied heavy hydrocarbons, only a dry residue, consisting only of low fractions can be obtained, which will no longer be removed, plus gases that can be extremely hazardous to health will be released. And chemical solutions can permanently poison underground waters, which will entail the death of all flora on the surface.

\section{About the method of the microbiological enhancement oil recovery (MEOR)}

This method of exposure is of two types:

1. Method of the biostimulation - injecttion of nutrients for the development of populations of microorganisms already existed in the oil reservoir.

2. Method of the bioaugmentation - injecti on of both microorganisms, and nutrients.

These bacteria are aerobic and anaerobic, in the process of their development and life, they liquefy hydrocarbons, absorbing some of them and emitting gases.

Also, their colonies, growing, form biofilms, reducing the possibility of water crossflows, therefore this method is effective when applied in heavily flooded fields.

In comparison with the methods mentioned earlier, it is environmentally friendly, and this is one of the most important advantages today.

To implement it, there is no need to purchase additional equipment, you can simply use the existing one.

Bacterial nutrients can be food waste such as milk serum and molasses.

* Corresponding author: o.kuderinova@gmail.com 


\section{Data about the characteristics of deposits, where the microbiological method was implemented}

In order to derive general characteristics for the use of the above-mentioned method, articles about deposits were studied, where it had already been tested.

These are the projects of San Andres, Queen Sand, Tupunyato Refugio, Huabey, Xinjian [1], Norne [2], Romashkinskoe [3], Pirallahi [4, 5], Lisbon, NovoElkhovskoe, Pervomayskoe, Ersubaikinskoe, Berezovskoe, Sterrap [6], White Tiger [7], Bokor [8].

San Andres Project (U.S.A.): it was discovered in 1945, was produced by solution gas drive until the waterflood was started in 1967.

The Microbial EOR started in October 1994 oil in place was $239 \mathrm{bbls} / \mathrm{ac}-\mathrm{ft}$ with an oil saturation of $41 \%$. Current formation pressure is estimated at 1,000 psi. Rock properties are relatively inhospitable to microbes. The low $1.7 \mathrm{md}$ average horizontal permeability would normally be indicative of pore throat sizes well below what microbes could enter. Reservoir temperature at $115^{\circ} \mathrm{F}$ is ideal for microbe growth. Average production per well is 14 barrels of oil per day at a $91 \%$ water cut.

After 19 months of microbe treating by $10 \%$ or 40 barrels per day are received.

Queen Sand Project (U.S.A.): it was discovered in 1984 , this reservoir was quickly waterflooded due its very low solution gas content. Injection was begun in 1990 and oil production increased quickly from 200 to 2,500 barrels per day. This rate continued until late 1991 when a rapid decline began.

The Microbial EOR started in August 1992, oil in place was 758 bbls/ac- $\mathrm{ft}$ with an oil saturation of $56 \%$. Rock properties are generally favorable for microbe colonization. Average permeability is $13 \mathrm{md}$ with an upper limit of $300 \mathrm{md}$ and provides adequate pore throat size for microbes to colonize. Additional permeability developed by fracture treatments with 60,000 gallons and 135,000 pounds of sand on initial completion provide excellent porous media for microbe colonization. Reservoir temperature at $110^{\circ} \mathrm{F}$ is ideal for microbe growth. Average production per well is $42 \mathrm{BOPD}$ at $75 \%$ water cut.

After 24 months of treating by $43 \%$ or 300 barrels per day are received.

Tupungato-Refúgio Project (Argentina): it was discovered in 1930. The field was produced by a combination of solution gas drive, water drive and waterflood.

Microbial EOR was started on one well in June 1994 and on the other two wells in March 1995. At the start of the project oil in place was $625 \mathrm{bbls} / \mathrm{ac}-\mathrm{ft}$ with an oil saturation of $47 \%$ and a gas saturation of $10 \%$. The wells are on approximately 42 acre spacing. Rock and fluid properties are all favorable for microbe colonization.

After 14 months of treating by $29 \%$ or 60 BOPD are received.

Huabei Project (P. R. China): it contains seven wells located in the Huabei Petroleum Administration Bureau. The wells, in the later stage of being waterflooded, are scattered and not in the same reservoir. Therefore, while production data can be analyzed, reservoir performance cannot be determined for this grouping.

Microbial EOR started September 1994. The wells are rod pumped, with pumps set an average of 2,500 feet above the perforations. Reservoir and fluid parameters are all favorable for microbe growth.

Twelve months after the first treatment by $552 \%$ or 127 BOPD are received.

Xinjiang Project (P. R. China): it contains ten wells located in the Xinjiang Petroleum Administration Bureau. The wells, most in the later stage of being waterflooded, are scattered and not in the same reservoir. Therefore, while production data can be analyzed, reservoir performance cannot be determined for this grouping.

Microbial EOR started January 1995. The wells are rod pumped, with pumps set from 200 to as high as 6,000 feet above the perforations on one well. Reservoir and fluid parameters are all favorable for microbe growth.

Six months after the first treatment by $36 \%$ or 80 barrels per day are received.

Norne Project (Norway): was discovered in 1992, developing started in 1997 . Oil density is $862 \mathrm{~kg} / \mathrm{m}^{3}$, Sulphur cintent is $0,21 \%$, oil viscosity $-3 \mathrm{mPa} \cdot \mathrm{s}$.

The incremental oil recovery due to biofilm formation can be around $2 \%$ OOIP.

In conclusion, an incremental oil recovery of almost $15 \%$ OOIP can be achieved by the combined effect of surfactant, biofilm formation and the resulting microscopic fluid diversion. Each mechanism contributes to the overall effect, where the main contribution comes from production of surfactant.

Romashkinskoye Project (Russian Federation): production started in 1975, carbonate collector, depth 493-515 m, porosity $-9,8 \%$; pressure $-6-7 \mathrm{MPa}$, temperature of stratum $-17-23{ }^{\circ} \mathrm{C}$, oil density $903 \mathrm{~kg} / \mathrm{m}^{3}$, oil viscosity $-50-80 \mathrm{mPa} \cdot \mathrm{s}$, stratal water mineralization $40-60 \mathrm{~g} / \mathrm{l}$.

The studying of the implementation of the Microbial EOR started in 1997. As the result $27 \%$ of residual oil can be recovered against $13 \%$ in the beginning.

Pirallahi Project (Republic of Azermaijan): the productiona started in 1902. Before Microbial implementation oil production in the 3-d quarter of 2009 comparing with the 1 -st decreased on $30 \%$, production rate was 0,39 .

Microbial EOR started in August 2009. For the well number 931 production increased from 0,7 tons per day up to 1,8 tons per day.

Lisbon Project (U.S.A.): North Carolina state. It was the first implementation of the Microbial EOR in 1954, which also was successful.

Novo-Yelkhovskoye, Pervomayskoye, Yersubakiyskoye, Berezovskoye Projects (Russian federation): they are operated by OJSC "TatNeft", water cut $90 \%$, sandstone collectors.

After Microbial EOR implementation the produation increased on 59,$8 ; 44,5 ; 9,3 ; 7,4$ thousand tons.

Sterrap Project (U.S.A.): Kansas State, depth of occurrence $-1600 \mathrm{~m}$, water cut $-95 \%$. 
After Microbial EOR implementation, the production increased on 50-55 thousand barrels.

White Tiger Project (Socialist republic of Vietnam): it is located in the shelf, it's flooded by sea water, salinity $35-37 \mathrm{~g} / 1$, reservoir temperature $120^{\circ} \mathrm{C}$, pressure $4 \mathrm{MPa}$.

After Microbial EOR implementation, the oil displacement efficiency amounted to $57.4 \%$. Using a combined physico-chemical and microbiological method increased oil displacement efficiency by $14.2 \%$.

Bokor Project (Malaysia): high viscosity crude (4 to $10 \mathrm{cp}$ ) and low oil specific gravity of $20^{\circ} \mathrm{API}$, low recovery factor in major reservoirs ranges from $19 \%$ to $25 \%$ of its original oil in place. Water depth of near 220 feet below msl; porosity - 15-32\%; permeability - 50$4000 \mathrm{mD}$; oil gravity $-19-22^{\circ}$ API in the shallower reservoirs $(1500-3000 \mathrm{Ft}$. ss $)$ to $37^{\circ} \mathrm{API}$ in the deep reservoirs $(6300 \mathrm{Ft}$. ss).

After Microbial EOR implementation over 5 months period significant increase of the oil production rate and reduction of water cut were observed. The average oil production rate for the period increases by $270 \mathrm{~b} / \mathrm{d}$, which is equivalent to $47 \%$ oil incremental.

Summarizing the data of these articles, we can draw the following conclusions:

Table 1. Range of characteristics for the application of the microbiological method of impact on oil deposits.

\begin{tabular}{|l|c|}
\hline \multicolumn{1}{|c|}{ Options } & Range \\
\hline Depth of occurrence, $\mathrm{m}$ & $180-2500$ \\
\hline Total thickness, $\mathrm{m}$ & $3,88-18,2$ \\
\hline Collector types & terrigenous, carbonate \\
\hline Open porosity, $\%$ & $7,9-24$ \\
\hline Permeability, $\mathrm{mcm}^{2}$ & $0,03-300$ \\
\hline Temperature, ${ }^{\circ} \mathrm{C}$ & $17-120$ \\
\hline Pressure, $\mathrm{MPa}$ & $0,344-7$ \\
\hline Density, $\mathrm{kg} / \mathrm{m}^{3}$ & $830-914$ \\
\hline Water type & calcium chloride \\
\hline $\begin{array}{l}\text { Mineralization of } \\
\text { groundwater, } \mathrm{g} / \mathrm{l}\end{array}$ & $8-235$ \\
\hline
\end{tabular}

Taking into account everything, mentioned before, it is obvious, that MEOR is applicable for most of the existing fields.

\section{Low-profit deposits in Kazakhstan in accordance with the official data}

Naturally, in order to offer this method, first you need to find potential customers, collect information about fields, where it is already required, to implement methods for improving the oil recovery.

Although it is known, that most of the republic's oil reservoirs have been in operation for a long time, and, as written in the many articles, they are at a last stage of operation. The search on state sites gave the following results: relating to the Resolution of the Government of the Republic of Kazakhstan No. 449 dated June 27, 2019, and also the estimates of the independent experts we receive the following table.
Table 2. List of low-profit fields.

\begin{tabular}{|c|c|c|}
\hline Field & Location & \begin{tabular}{|c|} 
Geological \\
reserves, mln tons
\end{tabular} \\
\hline \multicolumn{3}{|c|}{ 1. Containing high viscosity oils } \\
\hline $\begin{array}{l}\text { Temir district, } \\
\text { Aktobe region }\end{array}$ & $\begin{array}{l}\text { Temir district, } \\
\text { Aktobe region }\end{array}$ & $\begin{array}{l}\text { Temir district, } \\
\text { Aktobe region }\end{array}$ \\
\hline $\begin{array}{c}\text { Tupkaragan district, } \\
\text { Mangistau region }\end{array}$ & $\begin{array}{l}\text { Tupkaragan district, } \\
\text { Mangistau region }\end{array}$ & $\begin{array}{c}\text { Tupkaragan } \\
\text { district, Mangistau } \\
\text { region }\end{array}$ \\
\hline $\begin{array}{l}\text { Temir district, } \\
\text { Aktobe region }\end{array}$ & $\begin{array}{l}\text { Temir district, } \\
\text { Aktobe region }\end{array}$ & $\begin{array}{l}\text { Temir district, } \\
\text { Aktobe region }\end{array}$ \\
\hline $\begin{array}{l}\text { Isatai district, } \\
\text { Atyrau region }\end{array}$ & $\begin{array}{l}\text { Isatai district, } \\
\text { Atyrau region }\end{array}$ & $\begin{array}{l}\text { Isatai district, } \\
\text { Atyrau region }\end{array}$ \\
\hline \multicolumn{3}{|c|}{ 2. Low-income } \\
\hline East Akshabulak & $\begin{array}{l}\text { Syrdarya district, } \\
\text { Kyzylorda region }\end{array}$ & $\mathrm{n} / \mathrm{d}$. \\
\hline North Akshabulak & $\begin{array}{l}\text { Syrdarya district, } \\
\text { Kyzylorda region }\end{array}$ & $\mathrm{n} / \mathrm{d}$. \\
\hline Yeszhan & $\begin{array}{l}\text { Syrdarya district, } \\
\text { Kyzylorda region }\end{array}$ & $\mathrm{n} / \mathrm{d}$. \\
\hline Zhylankabak & $\begin{array}{l}\text { Zhylyoi district, } \\
\text { Atyrau region }\end{array}$ & $\mathrm{n} / \mathrm{d}$. \\
\hline Zholdybay & $\begin{array}{l}\text { Makat district, } \\
\text { Atyrau region }\end{array}$ & $\begin{array}{c}1,5 \\
\text { recov. }-297 \\
\text { thousand tons }\end{array}$ \\
\hline Kalzhan & $\begin{array}{l}\text { Syrdarya district, } \\
\text { Kyzylorda region }\end{array}$ & $\mathrm{n} / \mathrm{d}$. \\
\hline $\begin{array}{c}\text { South } \\
\text { Kamyskol }\end{array}$ & $\begin{array}{l}\text { Zhylyoi district, } \\
\text { Atyrau region }\end{array}$ & $\mathrm{n} / \mathrm{d}$. \\
\hline $\begin{array}{l}\text { Kokzhide } \\
\text { post-salt }\end{array}$ & $\begin{array}{l}\text { Temir district, } \\
\text { Aktobe region }\end{array}$ & $\begin{array}{c}50,9 \\
\text { on the } 1^{\text {st }} \text { Jan. } 2008 \\
\text { res.rec.res. }-12,5\end{array}$ \\
\hline Krykmyltyk & $\begin{array}{l}\text { Zhylyoi district, } \\
\text { Atyrau region }\end{array}$ & $\mathrm{n} / \mathrm{d}$. \\
\hline Tobearal & $\begin{array}{c}\text { Kurmangazy } \\
\text { district, Atyrau } \\
\text { region }\end{array}$ & $\mathrm{n} / \mathrm{d}$. \\
\hline \multicolumn{3}{|c|}{ 3. Waterflooded } \\
\hline Uzen & $\begin{array}{l}\text { Karakiya district, } \\
\text { Mangistau region }\end{array}$ & 1100 \\
\hline Kumkol & $\begin{array}{l}\text { Ulytau district, } \\
\text { Karaganda region }\end{array}$ & $\begin{array}{c}90 \\
280 \text { - geol., } \\
130 \text { - recov., } \\
15 \text { bln } \mathrm{m}^{3} \text { gas }\end{array}$ \\
\hline $\begin{array}{c}\text { East } \\
\text { Kumkol }\end{array}$ & $\begin{array}{c}\text { Ulytau district, } \\
\text { Karaganda region }\end{array}$ & $\mathrm{n} / \mathrm{d}$. \\
\hline Arman & $\begin{array}{l}\text { Mangistau district, } \\
\text { Mangystau region }\end{array}$ & 30 \\
\hline Karaarna & $\begin{array}{l}\text { Zhylyoi district, } \\
\text { Atyrau region }\end{array}$ & $\begin{array}{c}27,6 \\
25,4-\text { recov. }\end{array}$ \\
\hline $\begin{array}{c}\text { East } \\
\text { Kokarna }\end{array}$ & $\begin{array}{l}\text { Zhylyoi district, } \\
\text { Atyrau region }\end{array}$ & $\begin{array}{c}6,8 \\
4-\text { recov. }\end{array}$ \\
\hline Matin & $\begin{array}{l}\text { Makat district, } \\
\text { Atyrau region }\end{array}$ & 30 \\
\hline North Pridorozhnoe & $\begin{array}{c}\text { Beineu district, } \\
\text { Mangystau region }\end{array}$ & 30 \\
\hline \multicolumn{3}{|c|}{ 4. Irreplaceable (worked out) } \\
\hline Zhaksymay & $\begin{array}{l}\text { Temir district, } \\
\text { Aktobe region }\end{array}$ & 25 \\
\hline Shubarkuduk & $\begin{array}{l}\text { Temir district, } \\
\text { Aktobe region }\end{array}$ & $\mathrm{n} / \mathrm{d}$. \\
\hline
\end{tabular}

It turns out there are only 24 fields, at the same time there is an article by NOC Kazakhoil, which states that out of 45 fields they operate, at least two, Dossor and 
Makat, are among the oldest in terms of exploitation time [9].

In this paragraph, the abbreviation "n/d." appears for the first time, meaning "no data", i.e., unfortunately, this information is closed for public use. It will often meet further.

\section{Classification of the hydrocarbon deposits of the Republic of Kazakhstan}

Further searches for the necessary information led to even more interesting discoveries - the number of deposits, published on the Wikipedia site is 216 deposits, and those found by mentions in media publications for five months -269 .

The database in Excel format was compiled, which includes information on 289 fields, of which 269 are Kazakhstani and 20 foreign, where the microbiological method of stimulating the formation has already been introduced. As a result, we've got the following table:

Table 3. Comparison results.

\begin{tabular}{|c|c|c|}
\hline Deposit volume & $\begin{array}{c}\text { Known number of } \\
\text { deposits (site } \\
\text { Wiki2.org) }\end{array}$ & $\begin{array}{c}\text { Number of deposits } \\
\text { mentioned on the } \\
\text { Internet }\end{array}$ \\
\hline $\begin{array}{c}\text { Supergiant } \\
\text { (more than 5 bln } \\
\text { tons) }\end{array}$ & 1 & 2 \\
\hline $\begin{array}{c}\text { Giant (more than } \\
\text { 1 bln tons) }\end{array}$ & 3 & 3 \\
\hline $\begin{array}{c}\text { Large (from 100 } \\
\text { mln tons) }\end{array}$ & 11 & 29 \\
\hline $\begin{array}{c}\text { Medium (from } \\
\text { 10 mln tons) }\end{array}$ & 26 & 42 \\
\hline Others & more than 1000 & 40 \\
\hline No data & - & 153 \\
\hline
\end{tabular}

The difference is quite tangible. Especially considering that the second supergiant, the Kurmangazy field, discovered in Soviet times with reserves of 6,200 million tons of raw materials and then called Kulalinskaya, for some reason is only mentioned in Russian sources, although its estimated reserves are only slightly inferior to Kashagan, which is the largest in the world offshore field with reserves of 6,400 million tons.

It's needed to pay attention to the fact that there are no data on the geological reserves of 153 fields out of 269 , i.e. more than half of the known deposits are in operation without disclosing their characteristics.

Another classification was drawn up in accordance with a slightly different division by geological reserves [10].

Table 4. Second classification.

\begin{tabular}{|c|c|c|}
\hline Options & Q-ty & Deposits' names \\
\hline $\begin{array}{c}\text { Unique } \\
\text { deposits } \\
\text { (more than } \\
\text { 300 mln } \\
\text { tons of oil }\end{array}$ & & \\
$\begin{array}{c}\text { \&/ or more } \\
\text { than 300 }\end{array}$ & & $\begin{array}{c}\text { Bekturly, Darkhan, Kalamkas, } \\
\text { Karachaganak, Kashagan, Khvalynskoye, } \\
\text { Kurmangazy, Nursultan, Rakushechnoye- } \\
\text { sea, Tengiz, Uzen, Zhanazhol, Zhetybai }\end{array}$ \\
\hline
\end{tabular}

\begin{tabular}{|c|c|c|}
\hline $\begin{array}{l}\text { Options } \\
\text { bln } \mathrm{m}^{3} \text { of } \\
\text { gas) }\end{array}$ & Q-ty & Deposits' names \\
\hline 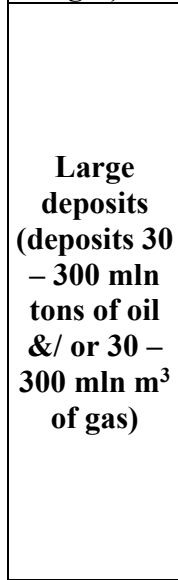 & 42 & \begin{tabular}{|c|} 
Akshabulak, Aktoty, Arman, Asar, Atash, \\
Bobek, Bozoba, Chinarevskoe, \\
Dosmukhambetovskoe, Dunga, East and \\
Central Prorva, East Tegen, Imashevskoye, \\
Isatayskoye, Kairan, Kalamkas-sea, \\
Karakuduk, Karaton-Koshkimbet, \\
Karazhanbas, Karpovsky Northern block, \\
Kenbai, Kenkiyak post-salt, Khasar, \\
Kokzhide post-salt, Korolevskoye, Kultuk \\
Mertvyi, Kumkol, Makhambet, Morskoye, \\
Mortuk, North Buzachi, North \\
Pridorozhnoye, North Truva, \\
Prigranichnoye, Rakushechnoye, \\
Shagyrly-Shomyshty, Tub-Karagan, \\
Turkmenoy, West Prorva, West \\
Terenozek, Zhambyl, Zhemchuzhina
\end{tabular} \\
\hline $\begin{array}{c}\begin{array}{c}\text { Medium } \\
\text { deposits }\end{array} \\
\text { (deposits } 5 \\
-30300 \\
\text { mln tons of } \\
\text { oil \&/ or } 5- \\
30 \text { mln m } \\
\text { of gas) }\end{array}$ & 32 & \begin{tabular}{|} 
Amangeldy, Ashhisay, Auezov, \\
Bolganmola, Borankol, East Kokarna, East \\
Zhagabulak, Kamenistoye, Kamenskoye, \\
Kansu, Karaarna, Karataikyz, Karatube, \\
Kemerkol, Komsomolskoye, Kozhasay, \\
Kulzhan, Kumsay suprasaline, Maibulak, \\
Masabay, North Akkar, North Karagie, \\
North Karamandybas, Nuraly, Oymasha, \\
Rozhkovskoye, Rozhkovskoye- \\
Fedorovsky block, South Gremyachinskoe, \\
Tyubedzhik, Zhaksymai, Zhambay South \\
Sea, Zhangurshi \\
\end{tabular} \\
\hline $\begin{array}{c}\text { Small } \\
\text { deposits } \\
\text { (deposits } 1 \\
-5 \text { mln tons } \\
\text { of oil \&/ or } \\
1-5 \text { mln } \\
\text { m }^{3} \text { of gas) } \\
\end{array}$ & 17 & $\begin{array}{c}\text { Airakty, Akingen, Aksai, Anabai, } \\
\text { Doschan, East Karaturun, Karabulak, } \\
\text { Kopa, Kyzyloyskoye, North Nuraly, North } \\
\text { Usharal, North-West Konys, } \\
\text { Rostoshinskoye, West Karabulak, West } \\
\text { Tuzkol, Zholdybai, Zhylandy }\end{array}$ \\
\hline $\begin{array}{l}\text { Very small } \\
\text { deposits } \\
\text { (less than } 1 \\
\text { mln tons of } \\
\text { oil \&/ or } \\
\text { less than } 1 \\
\text { blm } \text { m }^{3} \text { of } \\
\text { gas) }\end{array}$ & 12 & $\begin{array}{c}\text { Akkuduk, Arysskoe, Asanketen, } \\
\text { Ayrantakyr, Borkyldakty, East Kyzylzhar, } \\
\text { Koschagyl, Kulsary, Sagiz, Tuilis, Uytas, } \\
\text { West Sagiz }\end{array}$ \\
\hline No data & 153 & \begin{tabular}{|} 
Abay, Abylaikhan, Aiyrtau, Aksu- \\
Kenderly, Aktas, Aktobe, Akzhar, \\
Alatyube (East block), Alatyube, \\
Alibekmola, Alimbay, Altykol, Aryskum, \\
Arystan, Ashchiagar, Atambay-Sartube, \\
Auketayshagyl, Ayrankol, Baishonas, \\
Balgimbaev, Bazayskoye, Bekbike, \\
Bektas, Besbolek, Botakhan, Burmasha, \\
Daraimola, Daryinskoe, Dauletaly, Dossor, \\
East Akshabulak, East Akzhar, East \\
Gremyachinskoe, East Kumkol, East \\
Moldabek, East Normaul, East Ongar, East \\
Saztobe, East Uzen, East Zhetybay, \\
Eskene, Espelisay, Eszhan, Gran, \\
Gremyachinskoe, Gryadovoe, Kalzhan, \\
Karagan, Karagay, Karakuduk, Karatal, \\
Karaturun Marine, Kisimbai, Konys, \\
Korsak, Kenlyk, Kumisbek, Kyrykmyltyk, \\
Kyzylkiya, Laktybay, Makat, Makhat, \\
Maldybai, Matin, Munaily, Munaily-Mola, \\
Narmundanak, Naryn, North Akshabulak, \\
North Baklaniy, North Karazhanbas, North
\end{tabular} \\
\hline
\end{tabular}




\begin{tabular}{|c|c|c|}
\hline Options & Q-ty & Deposits' names \\
\hline & & $\begin{array}{l}\text { Kotyrtas, North Zholdybai, North-East } \\
\text { Karaturun, North-East Saztobe, North- } \\
\text { West Zhetybai, Nsanovskoe, Oktyabrskoe, } \\
\text { Okzhetpes, Ortalyk, Oryskazgan, } \\
\text { Pionerskoye, Port-Arthur, Pribrezhnoe, } \\
\text { Pridorozhnoe 1, Pridorozhnoe 2, } \\
\text { Pustynnoe, Ravninnoe, Rovnoe, } \\
\text { Sarsenbay, Satbayev site, Sazankurak, } \\
\text { Shagala, Shattyk, Shingiz, Shinzhir, } \\
\text { Shubarkuduk, Sinelnikovskoye, South } \\
\text { Alamuryn, South Kamyskol, South } \\
\text { Karatube, South Karaturun, South } \\
\text { Koshkar, South Kozha, South } \\
\text { Narmundanak, South Tagan, South } \\
\text { Tanatar, South Zhetybai, South-East } \\
\text { Bekturly, South-East Kamyshitovoye, } \\
\text { South-East Novobogatinskoe, South-East } \\
\text { Saztobe, South-West Dossor, South-West } \\
\text { Kamyshitovoye, South-West Tazhigali, } \\
\text { Tamdy, Tanatar, Tasbulat, Tastobe site, } \\
\text { Tasym, Tazhigali, Tenge, Tenteksor, } \\
\text { Teplovskoe, Tobearal, Tokarevskoe, } \\
\text { Tolegen, Tolkyn, Tortay, Tsentralnoye, } \\
\text { Tsyganovskoe, Tulpar, Ulyanovskoe, } \\
\text { Urikhtau, Usharal, Ushkultas, West Aktas, } \\
\text { West Novobogatinskoe, West Opak, West } \\
\text { Tenge, West Teplovskoe, Yuzhnaya } \\
\text { Rovnaya, Zaburunye, Zhanatalap, } \\
\text { Zhanatan, Zhanazhol, Zhangeldy, Zharty, } \\
\text { Zhenis site, Zhilankabak, Zhilankyr, } \\
\text { Zhubantam }\end{array}$ \\
\hline
\end{tabular}

Table 5. Classification of the deposits by location.

\begin{tabular}{|c|c|}
\hline Names of regions (districts) & Quantity \\
\hline 1. Aktobe region & 25 \\
\hline Baigany district & 7 \\
\hline Mugalzhar district & 6 \\
\hline Temir district & 10 \\
\hline Shalkar district & 2 \\
\hline 2. $\quad$ Atyrau region & 88 \\
\hline Zhylyoi district & 39 \\
\hline Isatay district & 13 \\
\hline Kyzylkogy district & 10 \\
\hline Kurmangazy district & 3 \\
\hline Makat district & 22 \\
\hline Makhambet district & \begin{tabular}{|l|}
1 \\
\end{tabular} \\
\hline 3. $\quad$ Zhambyl region & 5 \\
\hline Moyinkum district & 3 \\
\hline Talas district & 2 \\
\hline 4. West Kazakhstan region & 19 \\
\hline Baiterek district & 14 \\
\hline Burlinsky district & 1 \\
\hline Zhangalinsky district & 1 \\
\hline Kaztalovsky district & 1 \\
\hline Taskaly district & 2 \\
\hline 5. Karaganda region & 5 \\
\hline Ulytau district & 5 \\
\hline 6. Kyzylorda region & 18 \\
\hline Zhalagash district & 4 \\
\hline Syrdarya district & 13 \\
\hline Shiely district & 1 \\
\hline 7. Mangystau region & 65 \\
\hline Beyneu district & 12 \\
\hline
\end{tabular}

\begin{tabular}{|c|c|}
\hline Names of regions (districts) & Quantity \\
\hline Karakiya district & 36 \\
\hline Mangystau district & 11 \\
\hline Munaylinsky district & 1 \\
\hline Tupkaragan district & 5 \\
\hline 8. $\quad$ Turkestan region & 3 \\
\hline $\begin{array}{r}\text { No data about the exact regional location at the } \\
\text { district level }\end{array}$ & 3 \\
\hline $\begin{array}{l}\text { 9. Kazakhstani part of the Caspian Sea } \\
\text { shelf }\end{array}$ & 29 \\
\hline 10. No data on the exact geographic location & 12 \\
\hline
\end{tabular}

Over the period of independence of the Republic of Kazakhstan, i.e. since 1991, only 42 fields have been discovered: Kashagan, Aktoty, Kalamkas-sea, Kairan, Aiyrtau, Asanketen, Auezov, Ashisay, Borkyldakty, Gremyachinskoye Yuzhnoye, Doschan, Vostochny Zhagabulak, Zhambyl, Zhanatan, Zhemchuzhina, Karabulakulak, Zapadny Karaturun North-East, Kemerkol, Kokzhide post-salt, Kyzylzhar East, Laktybay, Narmundanak South, Novobogatinskoe West, Nuraly North, Border, Pridorozhnoe (Mangistau region), Rakushechnoye, Rakushechnoye-sea, Severnaya, Rozhkovskoe, Rostoshinskoye, Western Tuzkol, Tulpar, Uytas, Isataisskoye, Khazar, Khvalynskoye, Centralnoye, Chinarevskoye.

During its time in the USSR - 158 deposits.

Could not find data on the year of discovery of 69 deposits.

In addition, the data on licensed field operators are often hidden, for example, the owners of 85 fields are unknown.

For a more complete picture, the phase states of the collectors have been added to the resulting database, since we are primarily interested in oil deposits.

Table 6. Classification of the deposits by phase state.

\begin{tabular}{|c|c|}
\hline Phase state & Quantity \\
\hline Gas (g) & 24 \\
\hline Gas, condensate (gc) & 15 \\
\hline Gas, condensate, oil (gco) & 2 \\
\hline Gas and oil (go) & 16 \\
\hline Oil bituminous (ob) & 3 \\
\hline Oil and gas (og) & 34 \\
\hline Oil, gas, condensate (ogc) & 32 \\
\hline Oil (o) & 103 \\
\hline No data & 40 \\
\hline & 269 \\
\hline
\end{tabular}

\section{Additional information}

Already after writing the chapter of the dissertation, and this article, an additional source of information was found. It is published in Moscow in 2010 the report "Current state and development trends of the oil and gas complex of Turkmenistan and other Central Asian countries of the Near Abroad" [11], which is known only in the circles of oil specialists, but also not in the public domain.

It says, that there are 304 hydrocarbon deposits in Kazakhstan, i.e. even more than was found by me, and 
this, of course, also expanded the already existing picture of the data.

In it deposits are listed and described by basins.

Table 7. Classification of the deposits by basins.

\begin{tabular}{|c|c|c|}
\hline Basin name & Fields & Q-ty \\
\hline Volgo-Uralsky & $\begin{array}{c}1 \text { oil field, } \\
1 \text { oilgascondensate field }\end{array}$ & 2 \\
\hline Zaisansky & 1 gas field & 1 \\
\hline Caspian & $\begin{array}{c}11-\text { gas fields, } \\
6 \text { - gascondensate fields, } \\
98 \text { - oil fields, } \\
49 \text { - oilgas fields, } \\
15 \text { - oilgascondensate } \\
\text { fields }\end{array}$ & 179 \\
\hline $\begin{array}{l}\text { North Caucasian- } \\
\text { Mangyshlak }\end{array}$ & $\begin{array}{c}10-\text { gas fields, } \\
5 \text { - gascondensate fields, } \\
10-\text { oil fields, } \\
19-\text { oilgas fields, } \\
12-\text { oilgascondensate } \\
\text { fields } \\
\end{array}$ & 56 \\
\hline North Ustyurt & $\begin{array}{c}12 \text { - gas fields, } \\
4 \text { - gascondensate fields, } \\
3-\text { oil fields, } \\
13 \text { - oilgas fields } \\
\end{array}$ & 32 \\
\hline Turgay & $\begin{array}{c}2-\text { gas fields, } \\
2-\text { gascondensate fields, } \\
7-\text { oil fields, } \\
11-\text { oilgas fields, } \\
1 \text { - gascondensate fields }\end{array}$ & 23 \\
\hline Chu-Sarysu & $\begin{array}{c}9-\text { gas fields, } \\
2-\text { gascondensate fields }\end{array}$ & 11 \\
\hline
\end{tabular}

\section{Conclusions}

After carrying out of this work, the following conclusions are suggested:

1. Based on the data in the Table 1, it can be concluded that the microbiological impact method is applicable to the most hydrocarbon deposits of the republic, namely, referring to the Table 6 , at least 103, if not 172 .

2. Table 5 shows that Atyrau and Mangistau regions of Kazakhstan are leaders in terms of the number of deposits and the volume of hydrocarbons contained in them, but at the same time, it can also possible to see that 8 other Kazakhstan regions out of all 14 also have their own hydrocarbon raw materials. For example, we, the residents of the Karaganda region, did not know that we also have deposits of this type. Moreover, one of them, Kumkol, with initial reserves of 280 million tons of oil and 15 billion cubic meters of gas, in accordance with prepared classification belongs to the large one.

3. The oil and gas industry of our republic is one of the most closed to the public. But the disclosure and publication of these data, perhaps, will help to improve this situation.

\section{References}

1. F.L. Dietrich, F.G. Brown, Z.H. Zhou, M.A. Maure, Microb. EOR Technology Advancement: Case Studies of Successful Projects, SPE 36746 (1996)
2. S.D. Nielsen, Ph.D. thesis, MEOR - Adv. Reserv. Simulat., Technical University of Denmark (2010)

3. T.N. Nazina, A.E. Ivanova, G.F. Kandaurova, R.R. Ibatullin, S.S. Belyaev, M.V. Ivanov, Microb. Investig. of the Carbon. Collect. of the Romashkinskoye Oil F.: Backgr. St. bef. Test. a Biotechn. for the EOR (1998)

4. F.Y. Abdullayeva, S.S. Keldibayeva, Investig. of the MEOR applicap. in oilf. «Uzen», 4, 63 (2014)

5. A.M. Gasymly, N.I. Guseynova, F.Y. Abdullayeva, Exp. of Usage of Microbiol. Meth. of infl. on Oilfields of Azerbaijan (Case St. Pirallahi Oilfield) 4, 44-52 (2010)

6. G.Y. Sabakhova, K.R. Rafikova, M.R. Khisametdinov, Applicab. of the Microb. Infl. for Incr. of oil recov. (2015)

7. L.I. Svarovskaya, L.K. Altunina, The R. of Reserv. Microfl. in the Pr. of Oil Displacem. w. Comb. Phys.Chem. and Microb. M., Eurasian ChemTech Journal 4, 207-211 (2002)

8. M.G.A. Karim, M.A.H. Salim, Z.M. Zain, N.N. Talib, Microb. Enh. Oil Recov. (MEOR) Technology in Bokor Field, Sarawak, SPE 71125 (2001)

9. M. Ospanova, Nation. Oil Comp. KazakhOil (2009)

10. The Committee of the Geology and Subsoil Use (Ministry of Investment and Development of the Republic of Kazakhstan), Oil and gas fields of the Republic of Kazakhstan (2012)

11. M.N. Knepel, V.Y. Vysotskiy, T.G. Zargaryan et al., Cur. St. \& Dev. Tre-s of the $O \& G$ ind. of the Turkmenistan and oth. Cent. As. C-s of the N. Abr., 286 (2010)

12. Y. Malanchuk, V. Korniienko, L. Malanchuk, V. Zaiets, Res. into the Moist. Infl. on the Phys.-chem. T.-st. Char. in Bas. Q. of the Riv.-Vol. Reg., E3S WOC, 211 (2020)

13. Z. Malanchuk, V. Moshynskyi, Y. Malanchuk, V. Korniienko, M. Koziar, Res. of Res. into the Cont. of R. E. Mat. in M.-Made Phosphog. Dep., Key Engineering Materials 844, 77-87 (2020)

14. Z. Malanchuk, V. Korniyenko, Y. Malanchuk, A. Khrystyuk, M. Kozyar, Identif. of the Pr. of Hydromech. Extr. of Amb., E3S WoC 166 (2020) 